\title{
A type III porous-thermo-elastic problem with quasi-static microvoids
}

\author{
Noelia Bazarra • Alberto Castejón · José R. Fernández $\mathbb{B} \cdot$ Ramón Quintanilla
}

Received: 3 December 2020 / Accepted: 19 June 2021 / Published online: 3 July 2021

(C) The Author(s) 2021

\begin{abstract}
In this work we study, from the numerical point of view, a one-dimensional thermoelastic problem where the thermal law is of type III. Quasi-static microvoids are also assumed within the model. The variational formulation leads to a coupled linear system made of variational equations and it is written in terms of the velocity, the volume fraction and the temperature. Fully discrete approximations are introduced by using the finite element method and the backward Euler method. A discrete stability property and a priori error estimates are proved, deriving the linear convergence under adequate additional regularity. Finally, some numerical simulations are presented to demonstrate the accuracy of the approximation and the behavior of the solution.
\end{abstract}

N. Bazarra · A. Castejón · J. R. Fernández $(\bowtie)$

Universidade de Vigo, Departamento de Matemática

Aplicada I, 36310 Vigo, Spain

e-mail: jose.fernandez@uvigo.es

N. Bazarra

e-mail: nbazarra@uvigo.es

A. Castejón

e-mail: acaste@uvigo.es

R. Quintanilla

Departamento de Matemáticas, E.S.E.I.A.A.T.-U.P.C.

Colom 11, 08222 Terrassa, Barcelona, Spain

e-mail: ramon.quintanilla@upc.edu
Keywords Type III thermoelasticity · Quasi-static microvoids $\cdot$ Finite elements $\cdot$ A priori error analysis . Numerical simulations

Mathematics Subject Classification $65 \mathrm{M} 60$. 65M12 - 65M15 - 74F10 · 74F05 - 74K10

\section{Introduction}

After the proposition of Cosserat brothers [5] concerning micropolar elastic materials, a big deal was developed to generalize the elastic materials including microstructure. In fact, in the second part of the last century new proposals in this line were considered. The foundations of granular materials with intersticial voids were set down by Goodman and Cowin [15], and the theory of elastic solids with voids was introduced by Cowin and Nunziato $[6,7,30]$. It seems that the intention of these authors was to model the behavior of materials with small voids (or pores) distributed within them. Heat effects were also included in this theory [19-21]. It is worth saying that this theory has been assumed by the scientific community and the literature devoted to it is huge. In order to give a couple of examples, we can recall several contributions concerning the rate of decay for porous-thermoelasticity [2, 9-13, 22, 26, 31-34], and theirs numerical issues (see, for instance, [1, 14]). Applications of these materials are relevant in engineering and biology. 
In the decade of the 1990's, several theories were introduced to describe heat conduction. It seems that the main intention of the different authors was to overcome the paradox of the instantaneous propagation of the thermal waves obtained in the classical theory. In this paper, we are going to work with one of them. It is the one called type III that was introduced by Green and Naghdi [16-18]. In this case, a new unknown, the thermal displacement, is considered. The linear version of this theory has, as a particular subcase, the usual Fourier law. This new model proposes alternative couplings which cannot be present in the case of the Fourier theory. We believe that it is relevant to clarify the consequences of these new couplings. In this sense, we recall [27] and, even more [28], where the heat conduction does not propose dissipation of the thermal energy and it is usually called thermoelasticity without energy dissipation or type II theory. We also mention that a big difference in the time decay has been observed when we compare Fourier theory and type III theory with microtemperatures [23].

The idea to propose that a mechanism is quasistatic is as old as the mechanical sciences. In this situation, it is usually assumed that the change of a variable is so slow that the second time derivative of this variable can be neglected and it disappears from the equation. This could be a way to simplify the system of equations determining a process by means of a good approximation to the problem. It seems that Mosconi [29] was the first author proposing that the deformations of the microvoids can be considered so small to allow us to neglect its second-time derivative. In a recent paper [24], the one-dimensional problem of the porous elasticity with heat conduction of type II for quasi-static microvoids was analyzed from an analytical point of view and the exponential decay of solutions was obtained (see also [25]). Therefore, the same behavior holds in the case of the type III. In this paper, our aim is to continue the research started in $[24,25]$, providing the numerical analysis of a porouselastic problem arising in type III thermo-elasticity.

Therefore, since we concentrate our attention to the porous thermoelasticity of type III and we restrict ourselves to the one-dimensional setting, we assume that the domain of the material occupies the interval $[0, \ell], \ell>0$, and the evolution equations become (see [8]) $\rho \ddot{u}=\sigma_{x}, \quad J \ddot{\phi}=h_{x}+g, \quad T_{0} \dot{\eta}=q_{x}$,

where the constitutive equations are ${ }^{1}$

$$
\begin{aligned}
\sigma & =\mu u_{x}+b \phi-\beta \theta, \\
h & =\zeta \phi_{x}+l \alpha_{x}, \\
g & =-b u_{x}-\xi \phi+m \theta-\tau \dot{\phi}, \\
\eta & =c \theta+\beta u_{x}-m \phi, \\
q & =\kappa \theta_{x}+\kappa \alpha_{x}+l \phi_{x} .
\end{aligned}
$$

Therefore, assuming $T_{0}=1$ to make the calculations easier, the field equations are written as follows,

$$
\begin{aligned}
& \rho \ddot{u}=\mu u_{x x}+b \phi_{x}-\beta \theta_{x}, \\
& J \ddot{\phi}+\tau \dot{\phi}=\zeta \phi_{x x}+l \alpha_{x x}-b u_{x}-\xi \phi+m \theta, \\
& c \dot{\theta}=\kappa \alpha_{x x}+\kappa^{*} \theta_{x x}+l \phi_{x x}-\beta \dot{u}_{x}-m \dot{\phi} .
\end{aligned}
$$

In this system of equations, $u$ is the displacement, $\phi$ is the volume fraction, $\alpha$ is the thermal displacement, $\theta$ is the temperature, $\sigma$ is the stress, $h$ is the equilibrated stress, $g$ is the equilibrated body force, $\eta$ is the entropy, $q$ is the heat flux, $\rho$ is the mass density, $J$ is the product of the mass density by the equilibrated inertia, $T_{0}$ is the reference temperature, $c$ is the thermal capacity, $\kappa^{*}$ is the rate conductivity, $\kappa$ is the thermal conductivity, $\mu$ is the elasticity, $\beta$ is the thermomechanical coupling coefficient and $b, \zeta, l, b, \xi, m$ are constitutive coefficients. We also note that $\tau$ determines the porous dissipation. Moreover, the time derivative is denoted by a superposed dot for the first order or two superposed dots for the second order.

In the case that we assume that the deformations of the microvoids are quasi-static, we have that $\ddot{\phi} \approx 0$ and therefore we obtain the following linear system [24]:

$$
\begin{aligned}
\rho \ddot{u}= & \mu u_{x x}+b \phi_{x}-\beta \theta_{x}, \\
\tau \dot{\phi}= & \zeta \phi_{x x}+l \alpha_{x x}-b u_{x}-\xi \phi+m \theta, \\
c \dot{\theta}= & \kappa \alpha_{x x}+\kappa^{*} \theta_{x x}+l \phi_{x x}-\beta \dot{u}_{x} \\
& -\frac{m}{\tau}\left(\zeta \phi_{x x}+l \alpha_{x x}-b u_{x}-\xi \phi+m \theta\right) .
\end{aligned}
$$

It is worth recalling that the existence and time decay of the solutions have been studied recently [24]. In this paper, we study numerically the one-dimensional

\footnotetext{
$\overline{{ }^{1} \text { In this paper }}$ we assume porous dissipation.
} 
quasi-static system for the porous thermoelasticity of type III. To be precise, we will introduce a fully discrete approximation by using the implicit Euler scheme and the finite element method to approximate the time derivatives and the spatial domain, respectively. Then, we will prove a discrete stability property and a priori error estimates, which will lead to the linear convergence of the approximations assuming some additional regularity conditions. Finally, we will present some numerical simulations to show the efficiency of the approximation and the behavior of the solution.

\section{The thermomechanical problem}

As usual, the spatial variable is represented by $x \in(0, \ell)$, and for the time we use the notation $t \in$ $[0, T]$, being $T>0$ the final time. In order to simplify the writing, we will remove the dependence of our functions with respect to these two variables.

Since we assume that the rod is homogeneous and isotropic, the type III porous-thermo-elastic problem with quasistatic voids is written as follows (see [24]).

Problem P. Find the displacement field $u:[0, \ell] \times[0, T] \rightarrow \mathbb{R}$, the volume fraction $\phi:[0, \ell] \times$ $[0, T] \rightarrow \mathbb{R}$ and the temperature $\theta:[0, \ell] \times[0, T] \rightarrow \mathbb{R}$ such that field equations (1)-(3) are fulfilled in $(0, \ell) \times(0, T)$, and the following boundary and initial conditions are satisfied:

$$
\begin{aligned}
& u(x, 0)=u_{0}(x), \dot{u}(x, 0)=v_{0}(x) \quad \text { for a.e. } x \in[0, \ell], \\
& \phi(x, 0)=\phi_{0}(x) \quad \text { for a.e. } x \in[0, \ell], \\
& \alpha(x, 0)=\alpha_{0}(x), \theta(x, 0)=\theta_{0}(x) \quad \text { for a.e. } x \in[0, \ell], \\
& u(0, t)=u(\ell, t)=0 \quad \text { for a.e. } t \in(0, T), \\
& \phi(0, t)=\phi(\ell, t)=0 \quad \text { for a.e. } t \in(0, T), \\
& \theta(0, t)=\theta(\ell, t)=0 \quad \text { for a.e. } t \in(0, T) .
\end{aligned}
$$

In the rest of this paper we will assume the following conditions on the constitutive coefficients: $\rho>0, \quad \tau>0, \quad c>0, \quad \mu>0, \quad \zeta>0$, $\mu \xi>b^{2}, \quad \zeta \kappa>l^{2}, \quad \kappa^{*}>0$.

We note that, from conditions (10), we obtain that $\xi>0$ and $\kappa>0$.

The aim of this paper is to study numerically Problem $\mathrm{P}$ and so, we first introduce its variational formulation. Therefore, let us define the Hilbert space $Y=L^{2}(\Omega)$ with its scalar product $(\cdot, \cdot)$ and the corresponding norm $\|\cdot\|$. We also consider the variational space $V$ as follows,

$$
V=\left\{z \in H^{1}(0, \ell) ; z(0)=z(\ell)=0\right\} .
$$

The corresponding scalar product and norm are denoted by $(\cdot, \cdot)_{V}$ and $\|\cdot\|_{V}$, respectively.

Using integration by parts and the Dirichlet boundary conditions (7)-(9), we obtain the variational form of Problem $\mathrm{P}$, written in terms of the temperature $\theta$, the velocity field $v=\dot{u}$ and the volume fraction $\phi$.

Problem VP. Find the velocity field $v:[0, T] \rightarrow V$, the volume fraction $\phi:[0, T] \rightarrow V$ and the temperature $\theta:[0, T] \rightarrow V$ such that $v(0)=v_{0}, \phi(0)=\phi_{0}$, $\theta(0)=\theta_{0}$,and, for a.e. $t \in(0, T)$ and for all $w, r, s \in V$,

$$
\begin{aligned}
& \rho(\dot{v}(t), w)+\mu\left(u_{x}(t), w_{x}\right) \\
& \quad=b\left(\phi_{x}(t), w\right)-\beta\left(\theta_{x}(t), w\right) \\
& \tau(\dot{\phi}(t), r)+\zeta\left(\phi_{x}(t), r_{x}\right)+\xi(\phi(t), r) \\
& \quad=-l\left(\alpha_{x}(t), r_{x}\right)-b\left(u_{x}(t), r\right)+m(\theta(t), r), \\
& c(\dot{\theta}(t), s)+\kappa\left(\alpha_{x}(t), s_{x}\right)+\kappa^{*}\left(\theta_{x}(t), s_{x}\right) \\
& =-\left(l-\frac{m \zeta}{\tau}\right)\left(\phi_{x}(t), s_{x}\right)-\frac{m^{2}}{\tau}(\theta(t), s) \\
& \quad+\frac{l m}{\tau}\left(\alpha_{x}(t), s_{x}\right)-\beta\left(v_{x}(t), s\right)+\frac{m b}{\tau}\left(u_{x}(t), s\right) \\
& \quad+\frac{m \xi}{\tau}(\phi(t), s),
\end{aligned}
$$

where the displacement field $u$ and the thermal displacement $\alpha$ are obtained from the following equations:

$$
u(t)=\int_{0}^{t} v(s) d s+u_{0}, \quad \alpha(t)=\int_{0}^{t} \theta(s) d s+\alpha_{0} .
$$


Proceeding as in [24] we can prove the following existence and uniqueness result.

Theorem 1 Let assumptions (10) hold. Then, there exists a unique solution to Problem VP with the following regularity:

$$
u, \alpha \in C^{1}([0, T] ; V) \cap C^{2}([0, T] ; Y), \quad \phi \in C^{1}([0, T] ; V) .
$$

Moreover, this solution is asymptotically stable; that is, if we define the energy of the system $E$ as

$$
\begin{aligned}
E(t)= & \int_{0}^{\ell} \rho v^{2}(t)+\mu u_{x}^{2}(t)+c \theta^{2}(t)+\zeta \phi_{x}^{2}(t)+\xi \phi^{2}(t) \\
& +2 b u_{x}(t) \phi(t)+2 l \phi_{x}(t) \alpha_{x}(t)+\kappa \alpha_{x}^{2}(t) d x
\end{aligned}
$$

then

$$
E(t) \leq M e^{-\omega t} E(0) \quad \forall t \geq 0,
$$

for given positive constants $M$ and $\omega$.

\section{Numerical analysis of Problem VP: a priori error estimates}

For the spatial approximation of Problem VP, we assume that the interval $[0, \ell]$ is divided into $M$ subintervals $a_{0}=0<a_{1}<\cdots<a_{M}=\ell$ of length $h=a_{i+1}-a_{i}=\ell / M$. Then, in order to approximate the variational space $V$, we construct the finite dimensional space $V^{h} \subset V$ given by

$$
\begin{array}{r}
V^{h}=\left\{\xi^{h} \in C([0, \ell]) ; \xi_{\left[a_{i}, a_{i+1}\right]}^{h} \in P_{1}\left(\left[a_{i}, a_{i+1}\right]\right)\right. \\
\left.i=0, \ldots, M-1, \quad \xi^{h}(0)=\xi^{h}(\ell)=0\right\},
\end{array}
$$

where $P_{1}\left(\left[a_{i}, a_{i+1}\right]\right)$ represents the space of polynomials of degree less or equal to 1 in the subinterval $\left[a_{i}, a_{i+1}\right]$; i.e. the finite element space is composed of continuous and piecewise affine functions. Here, $h>0$ denotes the spatial discretization parameter. Moreover, we assume that the discrete initial conditions, denoted by $u_{0}^{h}, v_{0}^{h}, \phi_{0}^{h}, \alpha_{0}^{h}$ and $\theta_{0}^{h}$ are given by

$$
\begin{aligned}
u_{0}^{h}=P_{V^{h}} u_{0}, & v_{0}^{h}=P_{V^{h}} v_{0}, \quad \phi_{0}^{h}=P_{V^{h}} \phi_{0}, \\
\alpha_{0}^{h}=P_{V^{h}} \alpha_{0}, & \theta_{0}^{h}=P_{V^{h}} \theta_{0} .
\end{aligned}
$$

Here, $P_{V^{h}}$ is the classical finite element interpolation operator over $V^{h}$ (see [4]).
In order to provide the time discretization of Problem VP, we consider a uniform partition of the time interval $[0, T], \quad$ denoted by $0=t_{0}<t_{1}<\cdots<t_{N}=T$, with constant step size $k=$ $T / N$ and nodes $t_{n}=n k$ for $n=0,1, \ldots, N$. For a continuous function $z(t)$, we use the notation $z_{n}=z\left(t_{n}\right)$ and, for a sequence $\left\{z_{n}\right\}$, let $\delta z_{n}=\left(z_{n}-z_{n-1}\right) / k$ be the divided differences.

Therefore, using the backward Euler scheme in time, the fully discrete approximation of VP is the following.

Problem VP ${ }^{h k}$. Find the discrete velocity field $v^{h k}=\left\{v_{n}^{h k}\right\}_{n=0}^{N} \subset V^{h}$, the discrete volume fraction $\phi^{h k}=\left\{\phi_{n}^{h k}\right\}_{n=0}^{N} \subset V^{h}$ and the discrete temperature $\theta^{h k}=\left\{\theta_{n}^{h k}\right\}_{n=0}^{N} \subset V^{h}$ such that $v_{0}^{h k}=v_{0}^{h}, \phi_{0}^{h k}=\phi_{0}^{h}$, $\theta_{0}^{h k}=\theta_{0}^{h}$, and, for $n=1, \ldots, N$ and for all $w^{h}, r^{h}, s^{h} \in V^{h}$,

$$
\begin{aligned}
& \rho\left(\delta v_{n}^{h k}, w^{h}\right)+\mu\left(\left(u_{n}^{h k}\right)_{x}, w_{x}^{h}\right) \\
& =b\left(\left(\phi_{n}^{h k}\right)_{x}, w^{h}\right)-\beta\left(\left(\theta_{n}^{h k}\right)_{x}, w^{h}\right), \\
& \tau\left(\delta \phi_{n}^{h k}, r^{h}\right)+\zeta\left(\left(\phi_{n}^{h k}\right)_{x}, r_{x}^{h}\right)+\xi\left(\phi_{n}^{h k}, r^{h}\right) \\
& =-l\left(\left(\alpha_{n}^{h k}\right)_{x}, r_{x}^{h}\right)-b\left(\left(u_{n}^{h k}\right)_{x}, r^{h}\right)+m\left(\theta_{n}^{h k}, r^{h}\right), \\
& c\left(\delta \theta_{n}^{h k}, s^{h}\right)+\kappa\left(\left(\alpha_{n}^{h k}\right)_{x}, s_{x}^{h}\right)+\kappa^{*}\left(\left(\theta_{n}^{h k}\right)_{x}, s_{x}^{h}\right) \\
& =-\frac{m^{2}}{\tau}\left(\theta_{n}^{h k}, s^{h}\right)-\left(l-\frac{m \zeta}{\tau}\right)\left(\left(\phi_{n}^{h k}\right)_{x}, s_{x}^{h}\right) \\
& \quad+\frac{l m}{\tau}\left(\left(\alpha_{n}^{h k}\right)_{x}, s_{x}^{h}\right)-\beta\left(\left(v_{n}^{h k}\right)_{x}, s^{h}\right) \\
& \quad+\frac{m b}{\tau}\left(\left(u_{n}^{h k}\right)_{x}, s^{h}\right)+\frac{m \xi}{\tau}\left(\phi_{n}^{h k}, s^{h}\right),
\end{aligned}
$$

where the discrete displacement field $u_{n}^{h k}$ and the discrete thermal displacement $\alpha_{n}^{h k}$ are updated from the following equations:

$u_{n}^{h k}=k \sum_{j=1}^{n} v_{j}^{h k}+u_{0}^{h}, \quad \alpha_{n}^{h k}=k \sum_{j=1}^{n} \theta_{j}^{h k}+\alpha_{0}^{h}$.

By using conditions (10) and applying the well-known Lax Milgram lemma, it is easy to show that Problem $\mathrm{VP}^{h k}$ has a unique discrete solution.

First, we will prove the discrete stability of the solutions to Problem VP ${ }^{h k}$. 
Lemma 1 Under conditions (10), the sequences generated by Problem VPh , denoted by $\left\{u^{h k}, v^{h k}, \phi^{h k}, \alpha^{h k}, \theta^{h k}\right\}$, satisfy the following estimate:

$\left\|v_{n}^{h k}\right\|^{2}+\left\|\left(u_{n}^{h k}\right)_{x}\right\|^{2}+\left\|\phi_{n}^{h k}\right\|^{2}+\left\|\left(\alpha_{n}^{h k}\right)_{x}\right\|^{2}+\left\|\theta_{n}^{h k}\right\|^{2} \leq C$,

where the positive constant $C$ is independent of discretization parameters $h$ and $k$.

Proof Taking $w^{h}=v_{n}^{h k}$ in discrete variational equation (17) we have

$$
\begin{aligned}
& \rho\left(\delta v_{n}^{h k}, v_{n}^{h k}\right)+\mu\left(\left(u_{n}^{h k}\right)_{x},\left(v_{n}^{h k}\right)_{x}\right) \\
& \quad=b\left(\left(\phi_{n}^{h k}\right)_{x}, v_{n}^{h k}\right)-\beta\left(\left(\theta_{n}^{h k}\right)_{x}, v_{n}^{h k}\right),
\end{aligned}
$$

and therefore, keeping in mind that

$$
\begin{aligned}
& \left(\delta v_{n}^{h k}, v_{n}^{h k}\right) \geq \frac{1}{2 k}\left\{\left\|v_{n}^{h k}\right\|^{2}-\left\|v_{n-1}^{h k}\right\|^{2}\right\}, \\
& \left(\left(u_{n}^{h k}\right)_{x},\left(v_{n}^{h k}\right)_{x}\right) \geq \frac{1}{2 k}\left\{\left\|\left(u_{n}^{h k}\right)_{x}\right\|^{2}-\left\|\left(u_{n-1}^{h k}\right)_{x}\right\|^{2}\right\},
\end{aligned}
$$

it follows that

$$
\begin{aligned}
& \frac{\rho}{2 k}\left\{\left\|v_{n}^{h k}\right\|^{2}-\left\|v_{n-1}^{h k}\right\|^{2}\right\}+\frac{\mu}{2 k}\left\{\left\|\left(u_{n}^{h k}\right)_{x}\right\|^{2}-\left\|\left(u_{n-1}^{h k}\right)_{x}\right\|^{2}\right\} \\
& \leq C\left(\left\|\left(\phi_{n}^{h k}\right)_{x}\right\|^{2}+\left\|v_{n}^{h k}\right\|^{2}\right)-\beta\left(\left(\theta_{n}^{h k}\right)_{x}, v_{n}^{h k}\right) .
\end{aligned}
$$

Now, taking $r^{h}=\phi_{n}^{h k}$ in discrete variational equation (18) we find that

$$
\begin{aligned}
& \tau\left(\delta \phi_{n}^{h k}, \phi_{n}^{h k}\right)+\zeta\left(\left(\phi_{n}^{h k}\right)_{x},\left(\phi_{n}^{h k}\right)_{x}\right)+\xi\left(\phi_{n}^{h k}, \phi_{n}^{h k}\right) \\
& \quad=-l\left(\left(\alpha_{n}^{h k}\right)_{x},\left(\phi_{n}^{h k}\right)_{x}\right)-b\left(\left(u_{n}^{h k}\right)_{x}, \phi_{n}^{h k}\right)+m\left(\theta_{n}^{h k}, \phi_{n}^{h k}\right),
\end{aligned}
$$

and so, taking into account that

$$
\left(\delta \phi_{n}^{h k}, \phi_{n}^{h k}\right) \geq \frac{1}{2 k}\left\{\left\|\phi_{n}^{h k}\right\|^{2}-\left\|\phi_{n-1}^{h k}\right\|^{2}\right\},
$$

we obtain

$$
\begin{aligned}
& \frac{1}{2 k}\left\{\left\|\phi_{n}^{h k}\right\|^{2}-\left\|\phi_{n-1}^{h k}\right\|^{2}\right\}+C\left\|\left(\phi_{n}\right)_{x}\right\|^{2} \\
& \quad \leq C\left(\left\|\left(\alpha_{n}^{h k}\right)_{x}\right\|^{2}+\left\|\left(u_{n}^{h k}\right)_{x}\right\|^{2}+\left\|\theta_{n}^{h k}\right\|^{2}\right) .
\end{aligned}
$$

Finally, taking $s^{h}=\theta_{n}^{h k}$ as a test function in discrete variational equation (19) we get

$$
\begin{gathered}
c\left(\delta \theta_{n}^{h k}, \theta_{n}^{h k}\right)+\kappa\left(\left(\alpha_{n}^{h k}\right)_{x},\left(\theta_{n}^{h k}\right)_{x}\right)+\kappa^{*}\left(\left(\theta_{n}^{h k}\right)_{x},\left(\theta_{n}^{h k}\right)_{x}\right) \\
=-\frac{m^{2}}{\tau}\left(\theta_{n}^{h k}, \theta_{n}^{h k}\right)-\left(l-\frac{m \zeta}{\tau}\right)\left(\left(\phi_{n}^{h k}\right)_{x},\left(\theta_{n}^{h k}\right)_{x}\right) \\
+\frac{l m}{\tau}\left(\left(\alpha_{n}^{h k}\right)_{x},\left(\theta_{n}^{h k}\right)_{x}\right)-\beta\left(\left(v_{n}^{h k}\right)_{x}, \theta_{n}^{h k}\right) \\
+\frac{m b}{\tau}\left(\left(u_{n}^{h k}\right)_{x}, \theta_{n}^{h k}\right)+\frac{m \xi}{\tau}\left(\phi_{n}^{h k}, \theta_{n}^{h k}\right),
\end{gathered}
$$

and therefore, keeping in mind that

$$
\begin{aligned}
& \left(\delta \theta_{n}^{h k}, \theta_{n}^{h k}\right) \geq \frac{1}{2 k}\left\{\left\|\theta_{n}^{h k}\right\|^{2}-\left\|\theta_{n-1}^{h k}\right\|^{2}\right\}, \\
& \left(\left(\alpha_{n}^{h k}\right)_{x},\left(\theta_{n}^{h k}\right)_{x}\right) \geq \frac{1}{2 k}\left\{\left\|\left(\alpha_{n}^{h k}\right)_{x}\right\|^{2}-\left\|\left(\alpha_{n-1}^{h k}\right)_{x}\right\|^{2}\right\},
\end{aligned}
$$

we obtain

$$
\begin{aligned}
& \frac{c}{2 k}\left\{\left\|\theta_{n}^{h k}\right\|^{2}-\left\|\theta_{n-1}^{h k}\right\|^{2}\right\}+\frac{\kappa}{2 k}\left\{\left\|\left(\alpha_{n}^{h k}\right)_{x}\right\|^{2}-\left\|\left(\alpha_{n-1}^{h k}\right)_{x}\right\|^{2}\right\} \\
& \leq C\left(\left\|\left(\phi_{n}^{h k}\right)_{x}\right\|^{2}+\left\|\theta_{n}^{h k}\right\|^{2}+\left\|\left(\alpha_{n}^{h k}\right)_{x}\right\|^{2}+\left\|\left(u_{n}^{h k}\right)_{x}\right\|^{2}\right. \\
& \left.\quad+\left\|\phi_{n}^{h k}\right\|^{2}\right)-\beta\left(\left(v_{n}^{h k}\right)_{x}, \theta_{n}^{h k}\right) .
\end{aligned}
$$

Combining the previous estimates and using the equality

$$
-\beta\left(\left(v_{n}^{h k}\right)_{x}, \theta_{n}^{h k}\right)=\beta\left(v_{n}^{h k},\left(\theta_{n}^{h k}\right)_{x}\right),
$$

we find that

$$
\begin{aligned}
& \frac{\rho}{2 k}\left\{\left\|v_{n}^{h k}\right\|^{2}-\left\|v_{n-1}^{h k}\right\|^{2}\right\}+\frac{\mu}{2 k}\left\{\left\|\left(u_{n}^{h k}\right)_{x}\right\|^{2}-\left\|\left(u_{n-1}^{h k}\right)_{x}\right\|^{2}\right\} \\
& \quad+\frac{1}{2 k}\left\{\left\|\phi_{n}^{h k}\right\|^{2}-\left\|\phi_{n-1}^{h k}\right\|^{2}\right\}+\frac{c}{2 k}\left\{\left\|\theta_{n}^{h k}\right\|^{2}-\left\|\theta_{n-1}^{h k}\right\|^{2}\right\} \\
& \quad+\frac{\kappa}{2 k}\left\{\left\|\left(\alpha_{n}^{h k}\right)_{x}\right\|^{2}-\left\|\left(\alpha_{n-1}^{h k}\right)_{x}\right\|^{2}\right\} \\
& \leq C\left(\left\|v_{n}^{h k}\right\|^{2}+\left\|\left(\alpha_{n}^{h k}\right)_{x}\right\|^{2}+\left\|\left(u_{n}^{h k}\right)_{x}\right\|^{2}+\left\|\theta_{n}^{h k}\right\|^{2}+\left\|\phi_{n}^{h k}\right\|^{2}\right) .
\end{aligned}
$$

Summing up to $n$ we have

$$
\begin{aligned}
& \left\|v_{n}^{h k}\right\|^{2}+\left\|\left(u_{n}^{h k}\right)_{x}\right\|^{2}+\left\|\phi_{n}^{h k}\right\|^{2}+\left\|\theta_{n}^{h k}\right\|^{2}+\left\|\left(\alpha_{n}^{h k}\right)_{x}\right\|^{2} \\
& \leq C k \sum_{j=1}^{n}\left(\left\|v_{j}^{h k}\right\|^{2}+\left\|\theta_{j}^{h k}\right\|^{2}+\left\|\left(\alpha_{j}^{h k}\right)_{x}\right\|^{2}+\left\|\left(u_{j}^{h k}\right)_{x}\right\|^{2}\right. \\
& \left.\quad+\left\|\phi_{j}^{h k}\right\|^{2}\right)+C\left(\left\|v_{0}^{h}\right\|^{2}+\left\|\left(u_{0}^{h}\right)_{x}\right\|^{2}+\left\|\phi_{0}^{h}\right\|^{2}+\left\|\theta_{0}^{h}\right\|^{2}\right. \\
& \left.\quad+\left\|\left(\alpha_{0}^{h}\right)_{x}\right\|^{2}\right),
\end{aligned}
$$

and applying a discrete version of Gronwall's inequality (see, for instance, [3]), it follows the stability property. 
In the rest of this section, we will derive some a priori error estimates for the numerical errors. Therefore, we have the following.

Theorem 2 Let us denote by $(v, \phi, \theta)$ and $\left(v^{h k}, \phi^{h k}, \theta^{h k}\right)$ the solutions to problems VP and $V P^{h k}$, respectively. Under the conditions of Lemma 1 , then we have the following a priori error estimates, for all $\quad w^{h}=\left\{w_{j}^{h}\right\}_{j=0}^{N}, \quad r^{h}=\left\{r_{j}^{h}\right\}_{j=0}^{N} \quad$ and $s^{h}=\left\{s_{j}^{h}\right\}_{j=0}^{N} \subset V^{h}$,

$$
\begin{aligned}
& \max _{0 \leq n \leq N}\left\{\left\|v_{n}-v_{n}^{h k}\right\|^{2}+\left\|\left(u_{n}-u_{n}^{h k}\right)_{x}\right\|^{2}+\left\|\phi_{n}-\phi_{n}^{h k}\right\|^{2}\right. \\
& \left.+\left\|\theta_{n}-\theta_{n}^{h k}\right\|^{2}+\left\|\left(\alpha_{n}-\alpha_{n}^{h k}\right)_{x}\right\|^{2}\right\} \\
& \leq C k \sum_{j=1}^{N}\left(\left\|\dot{v}_{j}-\delta v_{j}\right\|^{2}+\left\|\left(\dot{u}_{j}-\delta u_{j}\right)_{x}\right\|^{2}+\left\|v_{j}-w_{j}^{h}\right\|_{V}^{2}\right. \\
& +\left\|\dot{\phi}_{j}-\delta \phi_{j}\right\|^{2}+\left\|\phi_{j}-r_{j}^{h}\right\|_{V}^{2}+\left\|\dot{\theta}_{j}-\delta \theta_{j}\right\|^{2} \\
& \left.+\left\|\left(\dot{\alpha}_{j}-\delta \alpha_{j}\right)_{x}\right\|^{2}+\left\|\theta_{j}-s_{j}^{h}\right\|_{V}^{2}\right) \\
& +C \max _{0 \leq n \leq N}\left(\left\|v_{n}-w_{n}^{h}\right\|^{2}+\left\|\phi_{n}-r_{n}^{h}\right\|^{2}+\left\|\theta_{n}-s_{n}^{h}\right\|^{2}\right) \\
& +C \frac{C}{k} \sum_{j=1}^{N-1}\left(\left\|v_{j}-w_{j}^{h}-\left(v_{j+1}-w_{j+1}^{h}\right)\right\|^{2}\right. \\
& +\left\|\phi_{j}-r_{j}^{h}-\left(\phi_{j+1}-r_{j+1}^{h}\right)\right\|^{2} \\
& \left.+\left\|\theta_{j}-s_{j}^{h}-\left(\theta_{j+1}-s_{j+1}^{h}\right)\right\|^{2}\right)+C\left(\left\|v_{0}-v_{0}^{h}\right\|^{2}\right. \\
& +\left\|\left(u_{0}-u_{0}^{h}\right)_{x}\right\|^{2}+\left\|\phi_{0}-\phi_{0}^{h}\right\|^{2}+\left\|\theta_{0}-\theta_{0}^{h}\right\|^{2} \\
& \left.+\left\|\left(\alpha_{0}-\alpha_{0}^{h}\right)_{x}\right\|^{2}\right)
\end{aligned}
$$

where we have denoted $\delta f_{j}=\left(f_{j}-f_{j-1}\right) / k$ for a function $f \in C([0, T] ; Y)$.

Proof First, we derive the estimates for the velocity field. By subtracting variational equation (11) at time $t=t_{n}$ for $w=w^{h} \in V^{h} \subset V$ and (17) it leads, for all $w^{h} \in V^{h}$

$$
\begin{aligned}
& \rho\left(\dot{v}_{n}-\delta v_{n}^{h k}, w^{h}\right)+\mu\left(\left(u_{n}-u_{n}^{h k}\right)_{x}, w_{x}^{h}\right)-b\left(\left(\phi_{n}-\phi_{n}^{h k}\right)_{x}, w^{h}\right) \\
& \quad+\beta\left(\left(\theta_{n}-\theta_{n}^{h k}\right)_{x}, w^{h}\right)=0,
\end{aligned}
$$

and therefore,

$$
\begin{aligned}
& \rho\left(\dot{v}_{n}-\delta v_{n}^{h k}, v_{n}-v_{n}^{h k}\right)+\mu\left(\left(u_{n}-u_{n}^{h k}\right)_{x},\left(v_{n}-v_{n}^{h k}\right)_{x}\right) \\
& \quad-b\left(\left(\phi_{n}-\phi_{n}^{h k}\right)_{x}, v_{n}-v_{n}^{h k}\right)+\beta\left(\left(\theta_{n}-\theta_{n}^{h k}\right)_{x}, v_{n}-v_{n}^{h k}\right) \\
& =\rho\left(\dot{v}_{n}-\delta v_{n}^{h k}, v_{n}-w^{h}\right)+\mu\left(\left(u_{n}-u_{n}^{h k}\right)_{x},\left(v_{n}-w^{h}\right)_{x}\right) \\
& \quad-b\left(\left(\phi_{n}-\phi_{n}^{h k}\right)_{x}, v_{n}-w^{h}\right)+\beta\left(\left(\theta_{n}-\theta_{n}^{h k}\right)_{x}, v_{n}-w^{h}\right) .
\end{aligned}
$$

Taking into account that

$$
\begin{aligned}
& \left(\dot{v}_{n}-\delta v_{n}^{h k}, v_{n}-v_{n}^{h k}\right)=\left(\dot{v}_{n}-\delta v_{n}, v_{n}-v_{n}^{h k}\right) \\
& \quad+\left(\delta v_{n}-\delta v_{n}^{h k}, v_{n}-v_{n}^{h k}\right) \\
& \quad \geq\left(\dot{v}_{n}-\delta v_{n}, v_{n}-v_{n}^{h k}\right) \\
& \quad+\frac{1}{2 k}\left\{\left\|v_{n}-v_{n}^{h k}\right\|^{2}-\left\|v_{n-1}-v_{n-1}^{h k}\right\|^{2}\right\}, \\
& \left(\left(u_{n}-u_{n}^{h k}\right)_{x},\left(v_{n}-v_{n}^{h k}\right)_{x}\right)=\left(\left(u_{n}-u_{n}^{h k}\right)_{x},\right. \\
& \left.\quad\left(\dot{u}_{n}-\delta u_{n}\right)_{x}\right)+\left(\left(u_{n}-u_{n}^{h k}\right)_{x},\left(\delta u_{n}-\delta u_{n}^{h k}\right)_{x}\right) \\
& \quad \geq\left(\left(u_{n}-u_{n}^{h k}\right)_{x},\left(\dot{u}_{n}-\delta u_{n}\right)_{x}\right) \\
& \quad+\frac{1}{2 k}\left\{\left\|\left(u_{n}-u_{n}^{h k}\right)_{x}\right\|^{2}-\left\|\left(u_{n-1}-u_{n-1}^{h k}\right)_{x}\right\|^{2}\right\}, \\
& \beta\left(\left(\theta_{n}-\theta_{n}^{h k}\right)_{x}, v_{n}-w^{h}\right)=-\beta\left(\theta_{n}-\theta_{n}^{h k},\left(v_{n}-w^{h}\right)_{x}\right), \\
& \text { we find that, for all } w^{h} \in V^{h}, \\
& \frac{\rho}{2 k}\left\{\left\|v_{n}-v_{n}^{h k}\right\|^{2}-\left\|v_{n-1}-v_{n-1}^{h k}\right\|^{2}\right\}+\beta\left(\left(\theta_{n}-\theta_{n}^{h k}\right)_{x},\right. \\
& \left.\quad v_{n}-v_{n}^{h k}\right)+\frac{\mu}{2 k}\left\{\left\|\left(u_{n}-u_{n}^{h k}\right)_{x}\right\|^{2}-\left\|\left(u_{n-1}-u_{n-1}^{h k}\right)_{x}\right\|^{2}\right\} \\
& \leq C\left(\left\|\dot{v}_{n}-\delta v_{n}\right\|^{2}+\left\|\left(\dot{u}_{n}-\delta u_{n}\right)_{x}\right\|^{2}+\left\|\left(\phi_{n}-\phi_{n}^{h k}\right)_{x}\right\|^{2}\right. \\
& \quad+\left\|v_{n}-v_{n}^{h k}\right\|^{2}+\left\|\theta_{n}-\theta_{n}^{h k}\right\|^{2}+\left\|v_{n}-w^{h}\right\|_{V}^{2} \\
& \left.\quad+\left\|\left(u_{n}-u_{n}^{h k}\right)_{x}\right\|^{2}+\left(\delta v_{n}-\delta v_{n}^{h k}, v_{n}-w^{h}\right)\right) .
\end{aligned}
$$

Now, we obtain the error estimates on the volume fraction. Thus, we subtract variational equation (12) at time $t=t_{n}$, for a test function $r=r^{h} \in V^{h} \subset V$, and discrete variational equation (18) to obtain

$$
\begin{aligned}
& \tau\left(\dot{\phi}_{n}-\delta \phi_{n}^{h k}, r^{h}\right)+\zeta\left(\left(\phi_{n}-\phi_{n}^{h k}\right)_{x}, r_{x}^{h}\right)+\xi\left(\phi_{n}-\phi_{n}^{h k}, r^{h}\right) \\
& \quad+l\left(\left(\alpha_{n}-\alpha_{n}^{h k}\right)_{x}, r_{x}^{h}\right)+b\left(\left(u_{n}-u_{n}^{h k}\right)_{x}, r^{h}\right) \\
& \quad-m\left(\theta_{n}-\theta_{n}^{h k}, r^{h}\right)=0
\end{aligned}
$$

and therefore, 


$$
\begin{aligned}
& \tau\left(\dot{\phi}_{n}-\delta \phi_{n}^{h k}, \phi_{n}-\phi_{n}^{h k}\right)+\zeta\left(\left(\phi_{n}-\phi_{n}^{h k}\right)_{x},\left(\phi_{n}-\phi_{n}^{h k}\right)_{x}\right) \\
& \quad+\xi\left(\phi_{n}-\phi_{n}^{h k}, \phi_{n}-\phi_{n}^{h k}\right)+l\left(\left(\alpha_{n}-\alpha_{n}^{h k}\right)_{x},\left(\phi_{n}-\phi_{n}^{h k}\right)_{x}\right) \\
& \quad+b\left(\left(u_{n}-u_{n}^{h k}\right)_{x}, \phi_{n}-\phi_{n}^{h k}\right)-m\left(\theta_{n}-\theta_{n}^{h k}, \phi_{n}-\phi_{n}^{h k}\right) \\
& =\tau\left(\dot{\phi}_{n}-\delta \phi_{n}^{h k}, \phi_{n}-r^{h}\right)+\zeta\left(\left(\phi_{n}-\phi_{n}^{h k}\right)_{x},\left(\phi_{n}-r^{h}\right)_{x}\right) \\
& \quad+\xi\left(\phi_{n}-\phi_{n}^{h k}, \phi_{n}-r^{h}\right)+l\left(\left(\alpha_{n}-\alpha_{n}^{h k}\right)_{x},\left(\phi_{n}-r^{h}\right)_{x}\right) \\
& \quad+b\left(\left(u_{n}-u_{n}^{h k}\right)_{x}, \phi_{n}-r^{h}\right)-m\left(\theta_{n}-\theta_{n}^{h k}, \phi_{n}-r^{h}\right) .
\end{aligned}
$$

Taking into account that

$$
\begin{aligned}
& \left(\dot{\phi}_{n}-\delta \phi_{n}^{h k}, \phi_{n}-\phi_{n}^{h k}\right)=\left(\dot{\phi}_{n}-\delta \phi_{n}, \phi_{n}-\phi_{n}^{h k}\right) \\
& \quad+\left(\delta \phi_{n}-\delta \phi_{n}^{h k}, \phi_{n}-\phi_{n}^{h k}\right) \\
& \quad \geq\left(\dot{\phi}_{n}-\delta \phi_{n}, \phi_{n}-\phi_{n}^{h k}\right) \\
& \quad+\frac{1}{2 k}\left\{\left\|\phi_{n}-\phi_{n}^{h k}\right\|^{2}-\left\|\phi_{n-1}-\phi_{n-1}^{h k}\right\|^{2}\right\}
\end{aligned}
$$

it follows that, for all $r^{h} \in V^{h}$,

$$
\begin{aligned}
\frac{1}{2 k}\{ & \left.\left\|\phi_{n}-\phi_{n}^{h k}\right\|^{2}-\left\|\phi_{n-1}-\phi_{n-1}^{h k}\right\|^{2}\right\} \\
\leq & C\left(\left\|\phi_{n}-\phi_{n}^{h k}\right\|^{2}+\left\|\dot{\phi}_{n}-\delta \phi_{n}\right\|^{2}+\left\|\phi_{n}-r^{h}\right\|_{V}^{2}\right. \\
& +\left\|\left(\alpha_{n}-\alpha_{n}^{h k}\right)_{x}\right\|^{2}+\left\|\left(u_{n}-u_{n}^{h k}\right)_{x}\right\|^{2}+\left\|\theta_{n}-\theta_{n}^{h k}\right\|^{2} \\
& \left.+\left(\delta \phi_{n}-\delta \phi_{n}, \phi_{n}-r^{h}\right)\right) .
\end{aligned}
$$

Finally, we get the error estimates on the temperature field. Thus, subtracting variational equation (13) at time $t=t_{n}$ for a test function $s=s^{h} \in V^{h} \subset V$ we have

$$
\begin{aligned}
& c\left(\dot{\theta}_{n}-\delta \theta_{n}^{h k}, s^{h}\right)+\kappa\left(\left(\alpha_{n}-\alpha_{n}^{h k}\right)_{x}, s_{x}^{h}\right) \\
& +\kappa^{*}\left(\left(\theta_{n}-\theta_{n}^{h k}\right)_{x}, s_{x}^{h}\right)-\frac{m^{2}}{\tau}\left(\theta_{n}-\theta_{n}^{h k}, s^{h}\right) \\
& +\left(l-\frac{m \zeta}{\tau}\right)\left(\left(\phi_{n}-\phi_{n}^{h k}\right)_{x}, s_{x}^{h}\right) \\
& \quad-\frac{l m}{\tau}\left(\left(\alpha_{n}-\alpha_{n}^{h k}\right)_{x}, s_{x}^{h}\right)+\beta\left(\left(v_{n}-v_{n}^{h k}\right)_{x}, s^{h}\right) \\
& -\frac{m b}{\tau}\left(\left(u_{n}-u_{n}^{h k}\right)_{x}, s^{h}\right) \\
& \quad-\frac{m \xi}{\tau}\left(\phi_{n}-\phi_{n}^{h k}, s^{h}\right)=0,
\end{aligned}
$$

and therefore, for all $s^{h} \in V^{h}$ it follows that

$$
\begin{aligned}
c\left(\dot{\theta}_{n}\right. & \left.-\delta \theta_{n}^{h k}, \theta_{n}-\theta_{n}^{h k}\right)+\kappa\left(\left(\alpha_{n}-\alpha_{n}^{h k}\right)_{x},\left(\theta_{n}-\theta_{n}^{h k}\right)_{x}\right) \\
& +\kappa^{*}\left(\left(\theta_{n}-\theta_{n}^{h k}\right)_{x},\left(\theta_{n}-\theta_{n}^{h k}\right)_{x}\right)-\frac{m^{2}}{\tau}\left(\theta_{n}-\theta_{n}^{h k},\right. \\
& \left.\theta_{n}-\theta_{n}^{h k}\right)+\left(l-\frac{m \zeta}{\tau}\right)\left(\left(\phi_{n}-\phi_{n}^{h k}\right)_{x},\left(\theta_{n}-\theta_{n}^{h k}\right)_{x}\right) \\
& -\frac{l m}{\tau}\left(\left(\alpha_{n}-\alpha_{n}^{h k}\right)_{x},\left(\theta_{n}-\theta_{n}^{h k}\right)_{x}\right) \\
& +\beta\left(\left(v_{n}-v_{n}^{h k}\right)_{x}, \theta_{n}-\theta_{n}^{h k}\right) \\
& -\frac{m b}{\tau}\left(\left(u_{n}-u_{n}^{h k}\right)_{x}, \theta_{n}-\theta_{n}^{h k}\right) \\
& -\frac{m \xi}{\tau}\left(\phi_{n}-\phi_{n}^{h k}, \theta_{n}-\theta_{n}^{h k}\right) \\
= & c\left(\dot{\theta}_{n}-\delta \theta_{n}^{h k}, \theta_{n}-s^{h}\right)+\kappa\left(\left(\alpha_{n}-\alpha_{n}^{h k}\right)_{x},\left(\theta_{n}-s^{h}\right)_{x}\right) \\
& +\kappa^{*}\left(\left(\theta_{n}-\theta_{n}^{h k}\right)_{x},\left(\theta_{n}-s^{h}\right)_{x}\right) \\
& -\frac{m^{2}}{\tau}\left(\theta_{n}-\theta_{n}^{h k}, \theta_{n}-s^{h}\right) \\
& \left.+\left(l-\frac{m \zeta}{\tau}\right)_{(}\left(\phi_{n}-\phi_{n}^{h k}\right)_{x},\left(\theta_{n}-s^{h}\right)_{x}\right) \\
& -\frac{l m}{\tau}\left(\left(\alpha_{n}-\alpha_{n}^{h k}\right)_{x},\left(\theta_{n}-s^{h}\right)_{x}\right) \\
& +\beta\left(\left(v_{n}-v_{n}^{h k}\right)_{x}, \theta_{n}-s^{h}\right) \\
& -\frac{m b}{\tau}\left(\left(u_{n}-u_{n}^{h k}\right)_{x}, \theta_{n}-s^{h}\right) \\
& -\frac{m \xi}{\tau}\left(\phi_{n}-\phi_{n}^{h k}, \theta_{n}-s^{h}\right) .
\end{aligned}
$$

Keeping in mind that

$$
\begin{aligned}
&\left(\dot{\theta}_{n}-\right.\left.\delta \theta_{n}^{h k}, \theta_{n}-\theta_{n}^{h k}\right)=\left(\dot{\theta}_{n}-\delta \theta_{n}, \theta_{n}-\theta_{n}^{h k}\right) \\
&+\left(\delta \theta_{n}-\delta \theta_{n}^{h k}, \theta_{n}-\theta_{n}^{h k}\right) \\
& \geq\left(\dot{\theta}_{n}-\delta \theta_{n}, \theta_{n}-\theta_{n}^{h k}\right) \\
&+\frac{1}{2 k}\left\{\left\|\theta_{n}-\theta_{n}^{h k}\right\|^{2}-\left\|\theta_{n-1}-\theta_{n-1}^{h k}\right\|^{2}\right\}, \\
&\left(\left(\alpha_{n}-\alpha_{n}^{h k}\right)_{x},\left(\theta_{n}-\theta_{n}^{h k}\right)_{x}\right)=\left(\left(\alpha_{n}-\alpha_{n}^{h k}\right)_{x},\right.\left.\left(\dot{\alpha}_{n}-\delta \alpha_{n}\right)_{x}\right)+\left(\left(\alpha_{n}-\alpha_{n}^{h k}\right)_{x},\left(\delta \alpha_{n}-\delta \alpha_{n}^{h k}\right)_{x}\right) \\
& \geq\left(\left(\alpha_{n}-\alpha_{n}^{h k}\right)_{x},\left(\dot{\alpha}_{n}-\delta \alpha_{n}\right)_{x}\right) \\
&+\frac{1}{2 k}\left\{\left\|\left(\alpha_{n}-\alpha_{n}^{h k}\right)_{x}\right\|^{2}-\left\|\left(\alpha_{n-1}-\alpha_{n-1}^{h k}\right)_{x}\right\|^{2}\right\}, \\
& \beta\left(\left(v_{n}-v_{n}^{h k}\right)_{x}, \theta_{n}-s^{h}\right)=-\beta\left(v_{n}-v_{n}^{h k},\left(\theta_{n}-s^{h}\right)_{x}\right), \\
& \beta\left(\left(v_{n}-v_{n}^{h k}\right)_{x}, \theta_{n}-\theta_{n}^{h k}\right)=-\beta\left(v_{n}-v_{n}^{h k},\left(\theta_{n}-\theta_{n}^{h k}\right)_{x}\right),
\end{aligned}
$$

we obtain, for all $s^{h} \in V^{h}$, 


$$
\begin{aligned}
\frac{c}{2 k}\{ & \left.\left\|\theta_{n}-\theta_{n}^{h k}\right\|^{2}-\left\|\theta_{n-1}-\theta_{n-1}^{h k}\right\|^{2}\right\} \\
& +\frac{\kappa}{2 k}\left\{\left\|\left(\alpha_{n}-\alpha_{n}^{h k}\right)_{x}\right\|^{2}-\left\|\left(\alpha_{n-1}-\alpha_{n-1}^{h k}\right)_{x}\right\|^{2}\right\} \\
& -\beta\left(v_{n}-v_{n}^{h k},\left(\theta_{n}-\theta_{n}^{h k}\right)_{x}\right) \\
\leq & C\left(\left\|\dot{\theta}_{n}-\delta \theta_{n}\right\|^{2}+\left\|\left(\dot{\alpha}_{n}-\delta \alpha_{n}\right)_{x}\right\|^{2}+\left\|\theta_{n}-\theta_{n}^{h k}\right\|^{2}\right. \\
& +\left\|\left(\alpha_{n}-\alpha_{n}^{h k}\right)_{x}\right\|^{2}+\left\|\phi_{n}-\phi_{n}^{h k}\right\|^{2}+\left\|\left(u_{n}-u_{n}^{h k}\right)_{x}\right\|^{2} \\
& +\left\|\left(\phi_{n}-\phi_{n}^{h k}\right)_{x}\right\|^{2}+\left\|\theta_{n}-s^{h}\right\|_{V}^{2}+\left\|v_{n}-v_{n}^{h k}\right\|^{2} \\
& \left.+\left(\delta \theta_{n}-\delta \theta_{n}^{h k}, \theta_{n}-s^{h}\right)\right) .
\end{aligned}
$$

Combining the above estimates, we find, for all $w^{h}, r^{h}, s^{h} \in V^{h}$,

$$
\begin{aligned}
& \frac{\rho}{2 k}\left\{\left\|v_{n}-v_{n}^{h k}\right\|^{2}-\left\|v_{n-1}-v_{n-1}^{h k}\right\|^{2}\right\} \\
& \quad+\frac{\mu}{2 k}\left\{\left\|\left(u_{n}-u_{n}^{h k}\right)_{x}\right\|^{2}-\left\|\left(u_{n-1}-u_{n-1}^{h k}\right)_{x}\right\|^{2}\right\} \\
& \quad+\frac{1}{2 k}\left\{\left\|\phi_{n}-\phi_{n}^{h k}\right\|^{2}-\left\|\phi_{n-1}-\phi_{n-1}^{h k}\right\|^{2}\right\} \\
& \quad+\frac{c}{2 k}\left\{\left\|\theta_{n}-\theta_{n}^{h k}\right\|^{2}-\left\|\theta_{n-1}-\theta_{n-1}^{h k}\right\|^{2}\right\} \\
& \quad+\frac{\kappa}{2 k}\left\{\left\|\left(\alpha_{n}-\alpha_{n}^{h k}\right)_{x}\right\|^{2}-\left\|\left(\alpha_{n-1}-\alpha_{n-1}^{h k}\right)_{x}\right\|^{2}\right\} \\
& \leq C\left(\left\|\dot{v}_{n}-\delta v_{n}\right\|^{2}+\left\|\left(\dot{u}_{n}-\delta u_{n}\right)_{x}\right\|^{2}+\left\|\left(\phi_{n}-\phi_{n}^{h k}\right)_{x}\right\|^{2}\right. \\
& \quad+\left\|v_{n}-v_{n}^{h k}\right\|^{2}+\left\|\phi_{n}-\phi_{n}^{h k}\right\|^{2}+\left\|\theta_{n}-\theta_{n}^{h k}\right\|^{2} \\
& \quad+\left\|v_{n}-w^{h}\right\|_{V}^{2}+\left\|\left(u_{n}-u_{n}^{h k}\right)_{x}\right\|^{2} \\
& \quad+\left(\delta v_{n}-\delta v_{n}^{h k}, v_{n}-w^{h}\right)+\left\|\dot{\phi}_{n}-\delta \phi_{n}\right\|^{2} \\
& \quad+\left\|\phi_{n}-r^{h}\right\|_{V}^{2}+\left\|\left(\alpha_{n}-\alpha_{n}^{h k}\right)_{x}\right\|^{2} \\
& \quad+\left(\delta \phi_{n}-\delta \phi_{n}, \phi_{n}-r^{h}\right)+\left\|\dot{\theta}_{n}-\delta \theta_{n}\right\|^{2} \\
& \quad+\left\|\left(\dot{\alpha}_{n}-\delta \alpha_{n}\right)_{x}\right\|^{2}+\left\|\theta_{n}-s^{h}\right\|_{V}^{2} \\
& \left.\quad+\left(\delta \theta_{n}-\delta \theta_{n}^{h k}, \theta_{n}-s^{h}\right)\right) .
\end{aligned}
$$

Multiplying these estimates by $k$ and summing up to $n$ we get, for all $\left\{w_{j}^{h}\right\}_{j=0}^{n},\left\{r_{j}^{h}\right\}_{j=0}^{n},\left\{s_{j}^{h}\right\}_{j=0}^{n} \subset V^{h}$,

$$
\begin{aligned}
\| v_{n}- & v_{n}^{h k}\left\|^{2}+\right\|\left(u_{n}-u_{n}^{h k}\right)_{x}\left\|^{2}+\right\| \phi_{n}-\phi_{n}^{h k} \|^{2} \\
& +\left\|\theta_{n}-\theta_{n}^{h k}\right\|^{2}+\left\|\left(\alpha_{n}-\alpha_{n}^{h k}\right)_{x}\right\|^{2} \\
\leq & C k \sum_{j=1}^{n}\left(\left\|\dot{v}_{j}-\delta v_{j}\right\|^{2}+\left\|\left(\dot{u}_{j}-\delta u_{j}\right)_{x}\right\|^{2}\right. \\
& +\left\|\left(\phi_{j}-\phi_{j}^{h k}\right)_{x}\right\|^{2}+\left\|v_{j}-v_{j}^{h k}\right\|^{2}+\left\|\phi_{j}-\phi_{j}^{h k}\right\|^{2} \\
& +\left\|\theta_{j}-\theta_{j}^{h k}\right\|^{2}+\left\|v_{j}-w_{j}^{h}\right\|_{V}^{2}+\left\|\left(u_{j}-u_{j}^{h k}\right)_{x}\right\|^{2} \\
& +\left(\delta v_{j}-\delta v_{j}^{h k}, v_{j}-w_{j}^{h}\right)+\left\|\dot{\phi}_{j}-\delta \phi_{j}\right\|^{2} \\
& +\left\|\left(\alpha_{j}-\alpha_{j}^{h k}\right)_{x}\right\|^{2}+\left\|\phi_{j}-r_{j}^{h}\right\|_{V}^{2}+\left\|\dot{\theta}_{j}-\delta \theta_{j}\right\|^{2} \\
& +\left(\delta \phi_{j}-\delta \phi_{j}, \phi_{j}-r_{j}^{h}\right)+\left\|\theta_{j}-s_{j}^{h}\right\|_{V}^{2} \\
& \left.+\left\|\left(\dot{\alpha}_{j}-\delta \alpha_{j}\right)_{x}\right\|^{2}+\left(\delta \theta_{j}-\delta \theta_{j}^{h k}, \theta_{j}-s_{j}^{h}\right)\right) \\
& +C\left(\left\|v_{0}-v_{0}^{h}\right\|^{2}+\left\|\left(u_{0}-u_{0}^{h}\right)_{x}\right\|^{2}+\left\|\phi_{0}-\phi_{0}^{h}\right\|^{2}\right. \\
& \left.+\left\|\theta_{0}-\theta_{0}^{h}\right\|^{2}+\left\|\left(\alpha_{0}-\alpha_{0}^{h}\right)_{x}\right\|^{2}\right) .
\end{aligned}
$$

Taking into account that

$$
\begin{aligned}
k \sum_{j=1}^{n} & \left(\delta v_{j}-\delta v_{j}^{h k}, v_{j}-w_{j}^{h}\right) \\
= & \sum_{j=1}^{n}\left(v_{j}-v_{j}^{h k}-\left(v_{j-1}-v_{j-1}^{h k}\right), v_{j}-w_{j}^{h}\right) \\
= & \left(v_{n}-v_{n}^{h k}, v_{n}-w_{n}^{h}\right)+\left(v_{0}^{h}-v^{0}, v_{1}-w_{1}^{h}\right) \\
\quad & +\sum_{j=1}^{n-1}\left(v_{j}-v_{j}^{h k}, v_{j}-w_{j}^{h}-\left(v_{j+1}-w_{j+1}^{h}\right)\right),
\end{aligned}
$$

where similar estimates are found for the corresponding terms involving the volume fraction and the temperature, we use a discrete version of Gronwall's inequality (see again [3]) and we conclude a priori error estimates (21).

As a consequence of the above estimates, we can obtain the convergence order of the finite element approximation, assuming suitable regularity conditions on the solution to Problem VP. Hence, we have the following (see [3] for details regarding the approximation of several terms).

Corollary 1 Under the assumptions of Theorem 2 and the additional regularity conditions: 


$$
\begin{aligned}
u \in & H^{3}(0, T ; Y) \cap W^{1, \infty}\left(0, T ; H^{2}(0, \ell)\right) \\
& \cap H^{2}\left(0, T ; H^{1}(0, \ell)\right), \\
\theta, \phi \in & H^{2}(0, T ; Y) \cap L^{\infty}\left(0, T ; H^{2}(0, \ell)\right) \\
& \cap H^{1}\left(0, T ; H^{1}(0, \ell)\right),
\end{aligned}
$$

we conclude the linear convergence of the approximations given by Problem VPhk that is, there exists a constant $C>0$, independent of parameters $h$ and $k$, such that

$$
\begin{aligned}
& \max _{0 \leq n \leq N}\left\{\left\|v_{n}-v_{n}^{h k}\right\|+\left\|\left(u_{n}-u_{n}^{h k}\right)_{x}\right\|+\left\|\phi_{n}-\phi_{n}^{h k}\right\|\right. \\
& \left.\quad+\left\|\theta_{n}-\theta_{n}^{h k}\right\|+\left\|\left(\alpha_{n}-\alpha_{n}^{h k}\right)_{x}\right\|\right\} \leq C(h+k) .
\end{aligned}
$$

\section{Numerical results}

In this section, we describe some numerical simulations to show the accuracy of the finite element approximation and the dependence on the solution with respect to the rate conductivity parameter. This last example will show the differences between type II and type III thermoelastic theories.

The numerical scheme was implemented on a 3.2 Ghz PC using MATLAB, and a typical run (using parameters $h=k=0.01$ ) took about $0.65 \mathrm{~s}$ of CPU time.

\subsection{First example: numerical convergence}

As a simpler example, in order to show the accuracy of the approximations we solve Problem $\mathrm{P}$ using the data:

$$
\begin{array}{ll}
T=1, & \ell=1, \quad \rho=1, \quad \mu=2, \quad b=1, \quad \beta=1, \\
\tau=1, & \zeta=2, \quad \xi=2, \quad l=1, \quad m=1, \quad c=1, \\
\kappa=2, & \kappa^{*}=1,
\end{array}
$$

the initial conditions, for $x \in(0,1)$,

$$
u_{0}(x)=v_{0}(x)=\phi_{0}(x)=\alpha_{0}(x)=\theta_{0}(x)=x(x-1) \text {, }
$$

the boundary conditions, for $t \in(0,1)$ and $x=0,1$,

$$
u(x, t)=\phi(x, t)=\alpha(x, t)=0,
$$

and the (artificial) supply terms, for $(x, t) \in(0,1) \times(0,1)$,

$$
\begin{aligned}
& F_{1}(x, t)=e^{t}(x(x-1)-2), \\
& F_{2}(x, t)=e^{t}(2 x+x(x-1)-5), \\
& F_{3}(x, t)=e^{t}(x(x-1)-2) .
\end{aligned}
$$

With the previous data and conditions, we can calculate the exact solution to Problem $P$ and it has the following form, for $(x, t) \in[0,1] \times[0,1]$ :

$$
u(x, t)=\phi(x, t)=\theta(x, t)=e^{t} x(x-1) .
$$

Thus, the approximation errors estimated by

$$
\begin{aligned}
& \max _{0 \leq n \leq N}\left\{\left\|v_{n}-v_{n}^{h k}\right\|+\left\|\left(u_{n}-u_{n}^{h k}\right)_{x}\right\|+\left\|\phi_{n}-\phi_{n}^{h k}\right\|\right. \\
& \left.\quad+\left\|\theta_{n}-\theta_{n}^{h k}\right\|+\left\|\left(\alpha_{n}-\alpha_{n}^{h k}\right)_{x}\right\|\right\}
\end{aligned}
$$

are shown in Table 1 for several values of the discretization parameters $h$ and $k$. Moreover, the evolution of the error depending on the parameter $h+$ $k$ is plotted in Fig. 1. We notice that the convergence of the algorithm is clearly proved, and the linear convergence, stated in Corollary 1, is achieved.

If we assume now that there are not supply terms, and we use the final time $T=20$, the following data

$$
\rho=1, \quad \ell=1, \quad b=4, \quad \beta=1, \quad \tau=1, \quad \zeta=2,
$$$$
\xi=1, \quad m=1, \quad c=1, \quad \kappa=4, \quad \kappa^{*}=1,
$$

and the initial conditions, for $x \in(0,1)$,

$$
u_{0}=v_{0}=\phi_{0}=x(x-1), \quad \alpha_{0}=\theta_{0}=0,
$$

taking the time discretization parameter $k=0.001$, the evolution in time of the discrete energy $E_{n}^{h k}$ given by

$$
\begin{aligned}
E_{n}^{h k}= & \int_{0}^{\ell} \rho\left(v_{n}^{h k}\right)^{2}+\mu\left(u_{n}^{h k}\right)_{x}^{2}+c\left(\theta_{n}^{h k}\right)^{2}+\zeta\left(\phi_{n}^{h k}\right)_{x}^{2} \\
& +\xi\left(\phi_{n}^{h k}\right)^{2}+\kappa\left(\alpha_{n}^{h k}\right)_{x}^{2}+2 l\left(\phi_{n}^{h k}\right)_{x}\left(\alpha_{n}^{h k}\right)_{x} d x,
\end{aligned}
$$

is plotted in Fig. 2 (in both natural and semi-log scales) for several values of the elastic coefficient $\mu$. As can be seen, it converges to zero and an exponential decay seems to be achieved for any value of the parameter. 
Table 1 Example 1: numerical errors for some $h$ and $k$

\begin{tabular}{llllllll}
\hline$h \downarrow k \rightarrow$ & 0.01 & 0.005 & 0.002 & 0.001 & 0.0005 & 0.0002 & 0.0001 \\
$1 / 2^{2}$ & 0.417612 & 0.416589 & 0.415997 & 0.415804 & 0.415708 & 0.415651 & 0.415633 \\
$1 / 2^{3}$ & 0.204233 & 0.203047 & 0.202390 & 0.202186 & 0.202087 & 0.202029 & 0.202010 \\
$1 / 2^{4}$ & 0.102405 & 0.100763 & 0.099979 & 0.099749 & 0.099641 & 0.099580 & 0.099561 \\
$1 / 2^{5}$ & 0.053109 & 0.051052 & 0.049939 & 0.049641 & 0.049516 & 0.049448 & 0.049427 \\
$1 / 2^{6}$ & 0.028948 & 0.026549 & 0.025301 & 0.024921 & 0.024744 & 0.024658 & 0.024634 \\
$1 / 2^{7}$ & 0.017463 & 0.014492 & 0.013055 & 0.012644 & 0.012452 & 0.012342 & 0.012308 \\
$1 / 2^{8}$ & 0.012482 & 0.008756 & 0.006993 & 0.006527 & 0.006320 & 0.006205 & 0.006169 \\
$1 / 2^{9}$ & 0.010642 & 0.006269 & 0.004061 & 0.003497 & 0.003263 & 0.003140 & 0.003102 \\
$1 / 2^{10}$ & 0.010076 & 0.005352 & 0.002741 & 0.002032 & 0.001749 & 0.001610 & 0.001570 \\
$1 / 2^{11}$ & 0.009923 & 0.005070 & 0.002225 & 0.001371 & 0.001016 & 0.000850 & 0.000805 \\
$1 / 2^{12}$ & 0.009884 & 0.004994 & 0.002058 & 0.001114 & 0.000686 & 0.000478 & 0.000425 \\
\hline
\end{tabular}

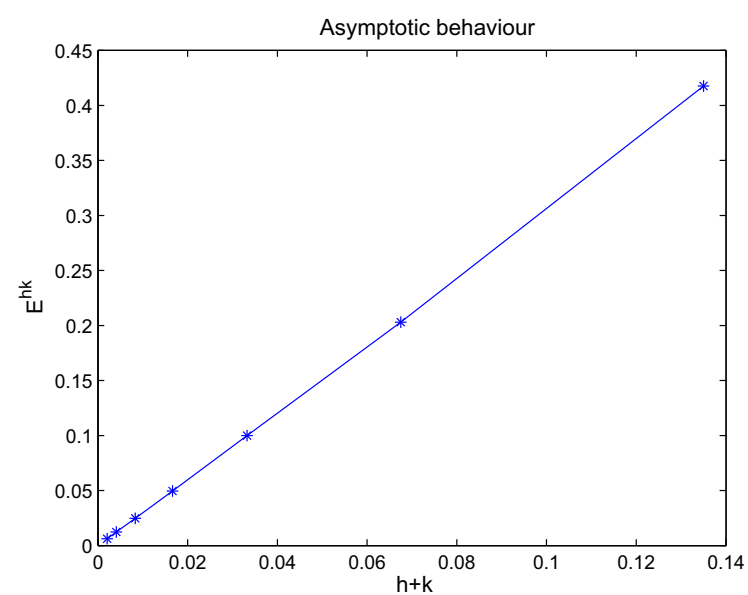

Fig. 1 Example 1: asymptotic constant error

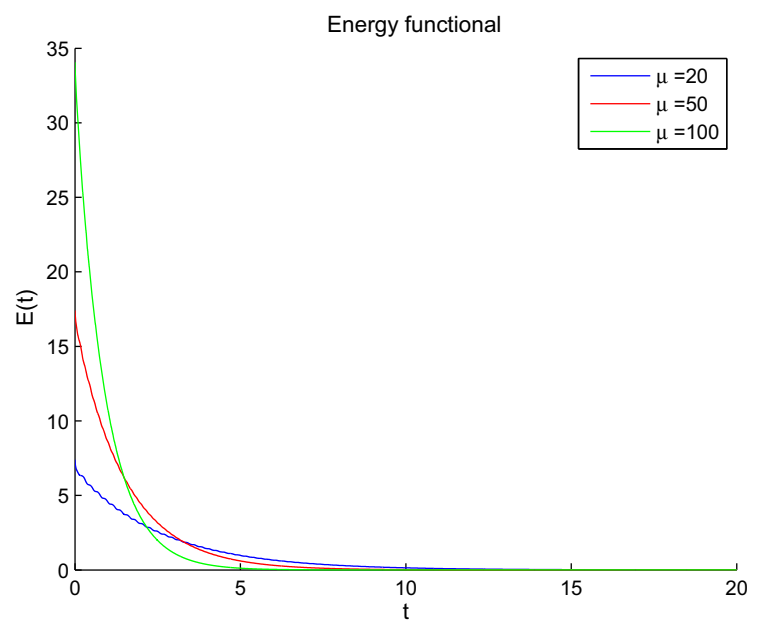

4.2 Second example: dependence on the rate conductivity parameter $\kappa^{*}$

As a second example, we study the dependence on the rate conductivity parameter $\kappa^{*}$. We note that the case $\kappa^{*}=0$ corresponds to the type II thermoelastic theory. We assume again that there are not supply terms, and we use final time $T=35$, the following data:

$\ell=1, \quad \rho=10, \quad \mu=4, \quad b=1, \quad \beta=3, \quad \tau=1$, $\eta=2, \quad \xi=6, \quad m=1, \quad c=1, \quad \kappa=10$,

and the initial conditions, for $x \in(0,1)$,

$$
u_{0}(x)=0, \quad \phi_{0}(x)=\alpha_{0}(x)=x(x-1) .
$$

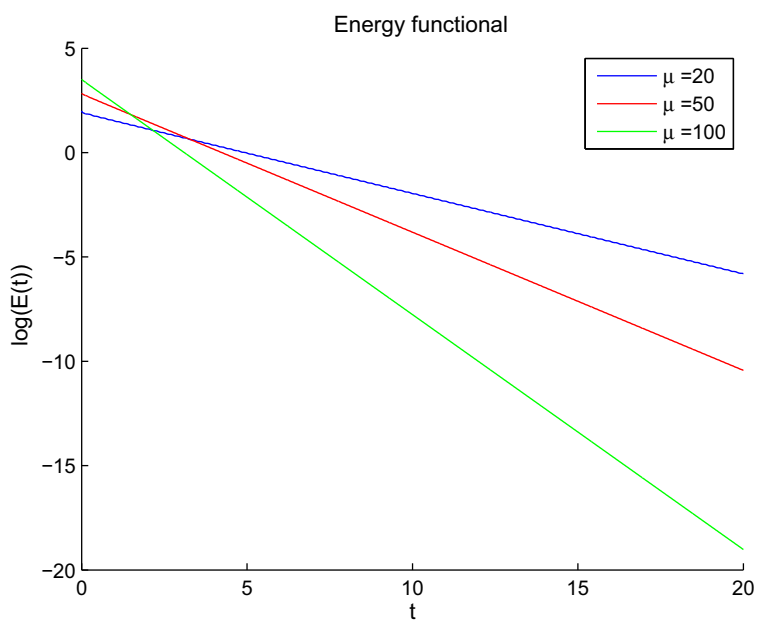

Fig. 2 Example 1: evolution in time of the discrete energy (natural and semi-log scales) for several values of $\mu$ 
Taking the discretization parameter $k=0.001$, in Fig. 3 the displacements, the porosity and the temperature are shown at final time. We can clearly observe that, when parameter $\kappa^{*}$ increases, the displacement field seems to converge to zero, meanwhile both the porosity and the temperature seem to change its shape.

Moreover, the evolution in time of the discrete energy $E_{n}^{h k}$ given above is plotted in Fig. 4 (in both natural and semi-log scales) for those values of $\kappa^{*}$. As can be seen, it always converges to zero and, after some time, an exponential decay seems to be achieved.

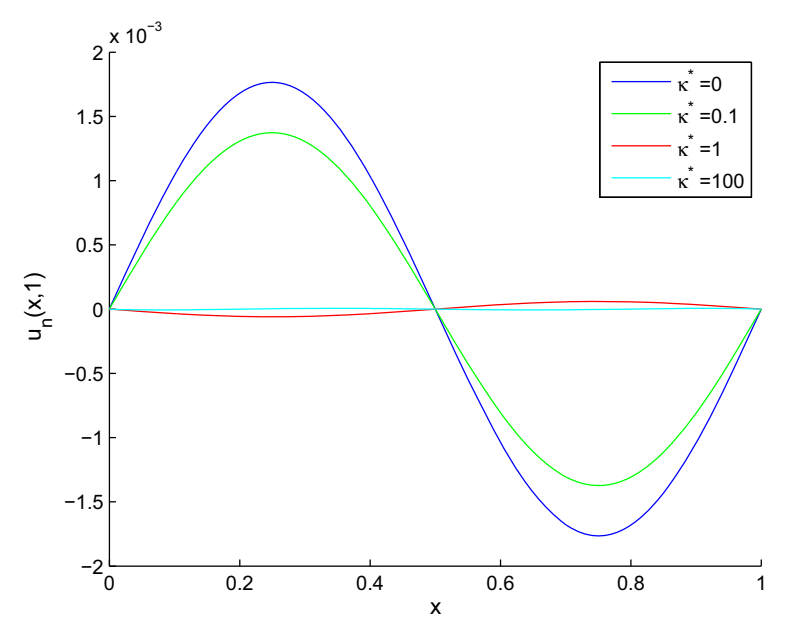

\section{Conclusions}

In this paper, we have studied, from the numerical point of view, a porous-elastic problem arising within the type II thermo-elastic theory. A fully discrete approximation has been introduced by using the finite element method and the implicit Euler scheme. A discrete stability property and some a priori error estimates have been proved, from which we have concluded the linear convergence of the approximation under suitable regularity conditions. Some numerical simulations have been performed to show the numerical convergence and the exponential decay of the discrete energy (Example 1). We have also included a comparison with the type II thermal law

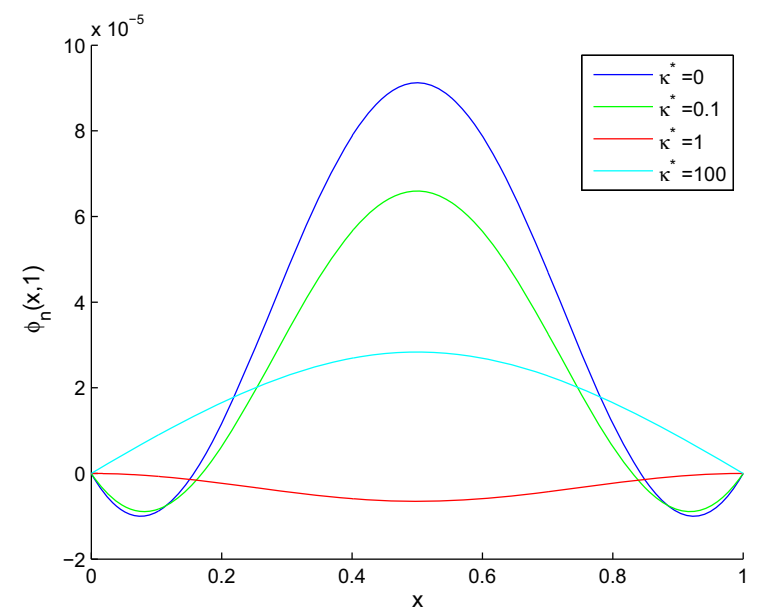

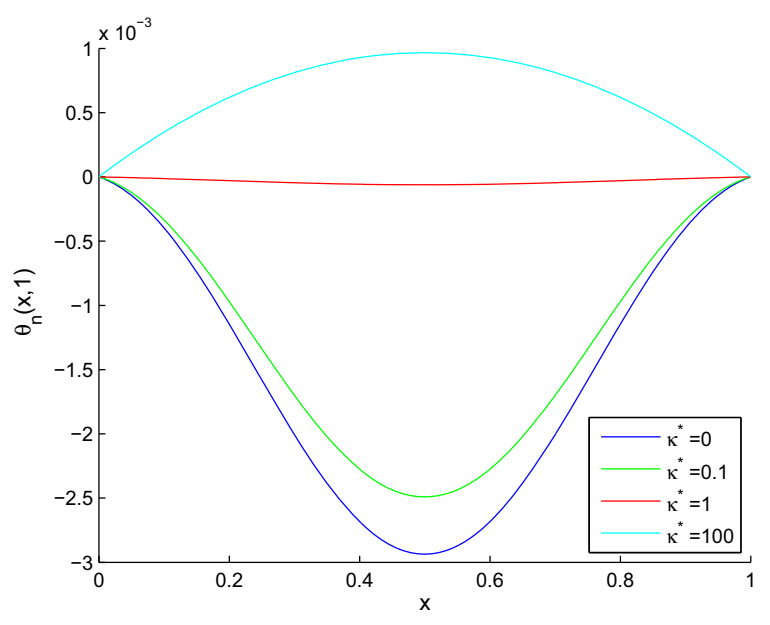

Fig. 3 Example 2: displacements (upper left), porosity (upper right) and temperature (lower) at final time for some values of $\kappa^{*}$ 

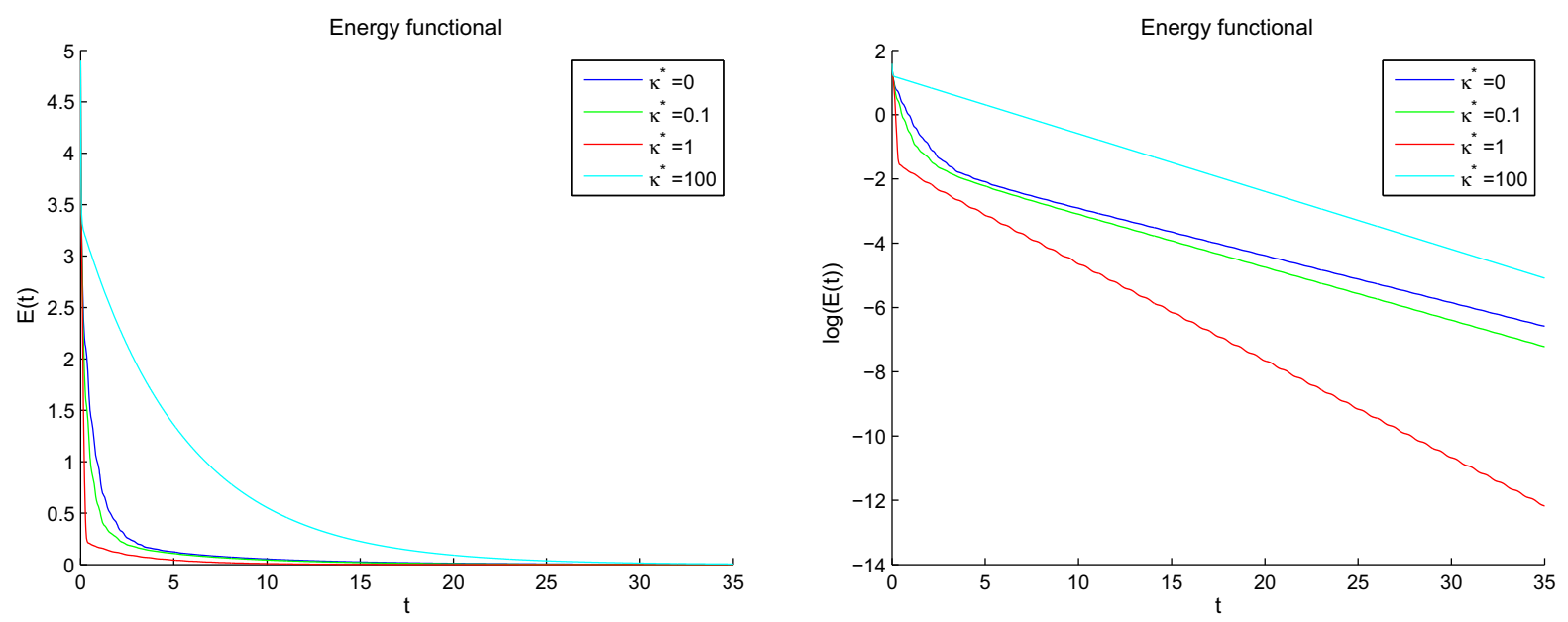

Fig. 4 Example 2: evolution in time of the discrete energy (natural and semi-log scales) for some values of $\kappa^{*}$

depending on the rate conductivity parameter (Example 2). Again, we have also demonstrated the exponential decay of the discrete energy for all the values of the parameter.

Acknowledgements The work of J.R. Fernández is partially supported by Ministerio de Ciencia, Innovación y Universidades under the research project PGC2018-096696-B-I00 (FEDER, UE). The work of R. Quintanilla is supported by the Ministerio de Ciencia, Innovación y Universidades under the research project "Análisis matemático aplicado a la termomecánica" (Ref. PID2019-105118GB-I00). We also point out that the funding for the open access charge is provided by Universidade de Vigo/CISUG.

\section{Declarations}

Conflict of interest The authors declare that they have no conflict of interest.

Open Access This article is licensed under a Creative Commons Attribution 4.0 International License, which permits use, sharing, adaptation, distribution and reproduction in any medium or format, as long as you give appropriate credit to the original author(s) and the source, provide a link to the Creative Commons licence, and indicate if changes were made. The images or other third party material in this article are included in the article's Creative Commons licence, unless indicated otherwise in a credit line to the material. If material is not included in the article's Creative Commons licence and your intended use is not permitted by statutory regulation or exceeds the permitted use, you will need to obtain permission directly from the copyright holder. To view a copy of this licence, visit http://creativecommons.org/licenses/by/4.0/.

\section{References}

1. Bazarra N, Fernández JR (2018) Numerical analysis of a contact problem in poro-thermoelasticity with microtemperatures. Z Angew Math Mech 98:1190-1209

2. Bazarra N, Fernández JR (2020) Quintanilla R Lord-Shulman thermoelasticity with microtemperatures. Appl Math Optim. https://doi.org/10.1007/s00245-020-09691-2

3. Campo M, Fernández JR, Kuttler KL, Shillor M, Viaño JM (2006) Numerical analysis and simulations of a dynamic frictionless contact problem with damage. Comput Methods Appl Mech Engrgy 196(1-3):476-488

4. Ciarlet PG (1993) Basic error estimates for elliptic problems. In: Ciarlet PG, Lions JL (eds) Handbook of numerical analysis, vol 2. North-Holland, Amsterdam, pp 17-351

5. Cosserat E, Cosserat F (1909) Théorie des corps déformables. Hermannn, Paris

6. Cowin SC (1985) The viscoelastic behavior of linear elastic materials with voids. J Elast 15:185-191

7. Cowin SC, Nunziato JW (1983) Linear elastic materials with voids. J Elast 13:125-147

8. De Cicco S, Diaco M (2002) A theory of thermoelastic materials with voids without energy dissipation. J Therm Stress 25:493-503

9. El-Naggar AM, Kishka Z, Abd-Alla AM, Abbas IA, AboDahab SM, Elsagheer M (2013) On the initial stress, magnetic field, voids and rotation effects on plane waves in generalized thermoelasticity. J Comput Theor Nanosci 10:1408-1417

10. Feng B (2018) Uniform decay of energy for a porous thermoelastic system with past history. Appl Anal 97:210-229

11. Feng B (2019) On the decay rates for a one-dimensional porous elasticity system with past history. Commun Pure Appl Anal 18:2905-2921

12. Feng B, Apalara TA (2019) Optimal decay for a porous elasticity system with memory. J Math Anal Appl 470:1108-1128 
13. Feng B, Yin M (2019) Decay of solutions for one-dimensional porous elasticity system with memory: the case of non-equal waves speed. Math Mech Solids 24:2361-2373

14. Fernández JR, Masid M (2017) A porous thermoelastic problem: an a priori error analysis and computational experiments. Appl Math Comput 305:117-135

15. Goodman MA, Cowin SC (1972) A continuum theory for granular materials. Arch Rat Mech Anal 44:249-266

16. Green AE, Naghdi PM (1992) On undamped heat waves in an elastic solid. J Therm Stress 15:253-264

17. Green AE, Naghdi PM (1993) Thermoelasticity without energy dissipation. J Elast 31:189-208

18. Green AE, Naghdi PM (1995) A unified procedure for construction of theories of deformable media. I. Classical continuum physics, II. Generalized continua, III. Mixtures of interacting continua. Proc R Soc Lond A 448, 335-356, 357-377, 379-388

19. Ieşan D (1986) A theory of thermoelastic materials with voids. Acta Mech 60:67-89

20. Ieşan D (2004) Thermoelastic models of continua. Springer, Berlin

21. Ieşan D, Nappa L (2004) Thermal stresses in plane strain of porous elastic bodies. Meccanica 39:125-138

22. Magaña A, Quintanilla R (2007) On the time decay of solutions in porous elasticity with quasi-static microvoids. J Math Anal Appl 331:617-630

23. Magaña A, Quintanilla R (2018) Exponential stability in type III thermoelasticity with microtemperatures. Zeits Ang Math Phys 69:129-1-129-8

24. Magaña A, Miranville A, Quintanilla R (2020) Exponential decay of solutions in type II porous-thermo-elasticity with quasi-static microvoids. J Math Anal Appl 492:124504

25. Magaña A, Quintanilla R (2021) Decay of quasi-static porous-thermo-elastic waves. Zeits Ang Math Phys 72:125
26. Marin M, Othman MIA, Abbas IA (2015) An extension of the domain of influence theorem for generalized thermoelasticity of anisotropic material with voids. J Comput Theor Nanosci 12:1594-1598

27. Miranville A, Quintanilla R (2019) Exponential stability in type III thermoelasticity with voids. Appl Math Lett 94:30-37

28. Miranville A, Quintanilla R (2020) Exponential decay in one-dimensional type II thermoviscoelasticity with voids. J Comput Appl Math 368:112573

29. Mosconi M (2005) A variational approach to porous elastic voids. Zeits Ang Math Phys 56:548-558

30. Nunziato JW, Cowin SC (1979) A nonlinear theory of elastic materials with voids. Arch Ration Mech Anal 72:175-201

31. Palani G, Abbas IA (2009) Free convection MHD flow with thermal radiation from an impulsively-started vertical plate. Nonlinear Anal Mod Control 14:73-84

32. Saeed T, Abbas IA, Marin M (2020) A GL model on thermo-elastic interaction in a poroelastic material using finite element method. Symmetry 12:488

33. Santos ML, Almeida Júnior DS (2017) On the porouselastic system with Kelvin-Voigt damping. J Math Anal Appl 445:498-512

34. Santos ML, Campelo ADS, Almeida Júnior DS (2017) On the decay rates of porous elastic systems. J Elast 127:79-101

Publisher's Note Springer Nature remains neutral with regard to jurisdictional claims in published maps and institutional affiliations. 